\title{
Accuracy of Vessel-Encoded Pseudocontinuous Arterial Spin-Labeling in Identification of Feeding Arteries in Patients with Intracranial Arteriovenous Malformations
}

\author{
S.L. Yu, R. Wang, R. Wang, S. Wang, Y.Q. Yao, D. Zhang, Y.L. Zhao, Z.T. Zuo, R. Xue, D.J.J. Wang, and J.Z. Zhao
}

\begin{abstract}
BACKGROUND AND PURPOSE: Identifying feeding arteries of intracranial AVMs is very important for preoperative evaluation. DSA remains the reference standard for diagnosis but is invasive. Our aim was to evaluate the diagnostic accuracy of vessel-encoded pseudocontinuous arterial spin-labeling in identifying feeding arteries of intracranial AVMs by using DSA as the criterion standard.
\end{abstract}

MATERIALS AND METHODS: Eighteen patients with AVMs were examined with vessel-encoded pseudocontinuous arterial spin-labeling and DSA. Three postlabeling delays (postlabeling delay $=1,1.3$, and 1.6 seconds) were applied in 6 patients, and a single postlabeling delay (1 second) was applied in the remainder. Perfusion-weighted images were decoded into individual vascular territories with standard and relative tagging efficiencies, respectively. The supply fraction of each feeding artery to the AVM was calculated. The within-subject ANOVA was applied to compare supply fractions acquired across 3 postlabeling delays. Receiver operating characteristic analysis curves were calculated to evaluate the diagnostic accuracy of vessel-encoded pseudocontinuous arterial spin-labeling for identifying the feeding arteries of AVMs.

RESULTS: There were no significant differences in supply fractions of the 3 major arteries to AVMs acquired with 3 postlabeling delays $(P>$ .05). For vessel-encoded pseudocontinuous arterial spin-labeling with standard labeling efficiencies, the area under the receiver operating characteristic analysis curve was 0.942 . The optimal cutoff of the supply fraction for identifying feeding arteries was $15.17 \%$, and the resulting sensitivity and specificity were $84.62 \%$ and $93.33 \%$, respectively. For vessel-encoded pseudocontinuous arterial spin-labeling with relative labeling efficiencies, the area under the receiver operating characteristic analysis curve was 0.957 . The optimal cutoff of the supply fraction was $11.73 \%$, which yielded an $89.74 \%$ sensitivity and $93.33 \%$ specificity.

CONCLUSIONS: The contribution fraction of each feeding artery of the AVM can be reliably estimated by using vessel-encoded pseudocontinuous arterial spin-labeling. Vessel-encoded pseudocontinuous arterial spin-labeling with either standard or relative labeling efficiencies offers a high level of diagnostic accuracy compared with DSA for identifying feeding arteries.

ABBREVIATIONS: ASL = arterial spin-labeling; $\mathrm{AUC}=$ area under the ROC curve; $\mathrm{LICA}=$ left internal carotid artery; $\mathrm{PLD}=$ postlabeling delay; $\mathrm{RICA}=$ right internal carotid artery; ROC = receiver operating characteristic analysis; VA = vertebral artery; VE-PCASL = vessel-encoded pseudocontinuous arterial spin-labeling

ntracranial AVMs are congenital vascular abnormalities with $\geq 1$ feeding artery and without a normal intervening capillary bed. The principal presentation of an AVM is parenchymal hem-

\footnotetext{
Received December 31, 2012; accepted after revision March 17, 2013.

From the Department of Neurosurgery (S.L.Y., Rong Wang, S.W., D.Z., Y.L.Z., J.Z.Z.), Beijing Tiantan Hospital, Capital Medical University, Beijing, China; State Key Laboratory of Brain and Cognitive Science (Rui Wang, Z.T.Z., R.X.), Beijing MRI Center for Brain Research, Institute of Biophysics, Chinese Academy of Sciences, Beijing, China; Department of Neurosurgery (Y.Q.Y.), Beijing Jishuitan Hospital, Peking University, Beijing, China; Department of Neurology (D.J.J.W.), University of California, Los Angeles, Los Angeles, California; and University of California, Los Angeles-Beijing Joint Center for Advanced Brain Imaging (R.X., Rui Wang, D.J.J.W.), Los Angeles, California and Beijing, China.

This work was supported by Natural Science Foundation of China of Tiantan Hospital (30830101), the National Institutes of Health (grants R01-MH080892, R01-NS081077 and R01-EB014922), the Ministry of Science and Technology of China (grant 2012CB825500), and the CAS Knowledge Innovation Project (grant KSCX2-YW-R-259).
}

orrhage, which accounts for $2 \%-4 \%$ of overall hemorrhagic strokes ${ }^{1}$ and confers significant morbidity and mortality. AVMs may increase in size and show vascular remodeling with time. The management of AVMs includes microsurgery, stereotactic radiosurgery, embolization, and multimodality treatments. Residual lesions may exist after microsurgery, though with a low incidence. ${ }^{2,3}$ Complete obliteration with stereotactic radiosurgery requires approximately $1-3$ years to complete, and a cure is not always achieved. ${ }^{4}$ For a large AVM, partial embolization is performed to make it amenable to further surgery or radiosurgery. ${ }^{5}$ The identification of feeding arteries of an AVM and calculation of corresponding contribution

Please address correspondence to Jizong Zhao, MD, Department of Neurosurgery, Beijing Tiantan Hospital, No. Six Tiantan Xili, Chongwen District, Beijing 100050, China; e-mail: zhaojz205@gmail.com

- Indicates open access to non-subscribers at www.ajnr.org

http://dx.doi.org/10.3174/ajnr.A3638 
fractions are helpful for standard microsurgical operations, in which the arterial feeders are generally occluded first, followed by excision of the nidus, and finally resection of the draining veins. ${ }^{6}$ Information about feeding arteries can also help in determining the sequence of arteries to be managed. A reliable, safe, and convenient imaging technology with the capability of identifying feeding arteries would be highly desirable for frequent examinations of AVMs.

DSA remains the criterion standard for the diagnosis and evaluation of intracranial AVMs. In our institution (Beijing Tiantan Hospital), it is usually performed just before the operation to identify feeding arteries and corresponding draining veins. As an invasive technique, DSA exposes both doctors and patients to the radiation of $\mathrm{x}$-rays and carries potential risks related to contrast agents and the experience of the operators. ${ }^{7}$ Although these results are rare, DSA may cause vessel injury, stroke, and even death, which is most concerning. ${ }^{8}$ Various MRA techniques have been explored for the evaluation of AVMs, such as 4D time-resolved dynamic MRA with or without contrast enhancement. ${ }^{9,10}$ Arterial spin-labeling (ASL) offers a method for quantifying regional CBF by using magnetically labeled arterial blood water as an endogenous tracer. ${ }^{11}$ While delayed arterial transit may cause underestimation of tissue perfusion in patients with stroke, the opposite effect-the rapid transit of labeled blood through arteriovenous shunts - can be exploited for direct visualization and, potentially, quantification of arteriovenous shunting. ${ }^{12,13}$ More recently, pseudocontinuous ASL has been introduced, which uses a train of discrete radio-frequency pulses to mimic flow-driven adiabatic inversion and combines the continuous superior signal-to-noise ratio of ASL and the higher tagging efficiency of pulsed ASL. ${ }^{14}$ Vessel-encoded pseudocontinuous arterial spin-labeling (VE-PCASL) is a time-efficient method for

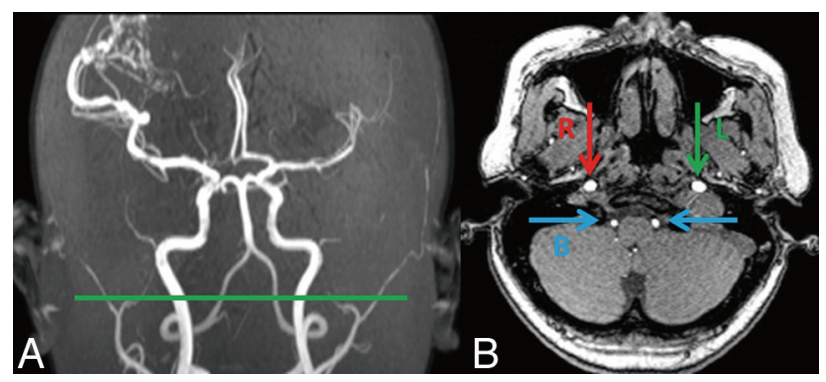

FIG 1. Labeling plane on the coronary $(A)$ and transverse $(B)$ MRA images. Arrows show RICA (R), LICA (L), and vertebrobasilar arteries (B).

Table 1: Modified Hadamard encoding matrix based on estimated relative labeling efficiencies of the 18 patients with AVMs

\begin{tabular}{ccccc}
\hline$A$ & -1.0000 & -1.0000 & -1.0000 & 1.0000 \\
& 1.0000 & 1.0000 & 1.0000 & 1.0000 \\
$-0.9200 \pm 0.083$ & $0.1600 \pm 0.186$ & $0.7100 \pm 0.340$ & 1.0000 \\
$0.9200 \pm 0.083$ & $-0.1600 \pm 0.186$ & $-0.7100 \pm 0.340$ & 1.0000 \\
$0.9700 \pm 0.105$ & $-0.5300 \pm 0.112$ & $0.9700 \pm 0.105$ & 1.0000 \\
$-0.9700 \pm 0.105$ & $0.5300 \pm 0.112$ & $-0.9700 \pm 0.105$ & 1.0000 \\
$\mathrm{~A}^{+}$ & $-0.1087 \pm 0.0312$ & $0.1087 \pm 0.0312$ & $-0.3067 \pm 0.0210$ & $0.3067 \pm 0.0210$ \\
$0.1125 \pm 0.0387$ & $-0.1125 \pm 0.0387$ & & \\
$-0.3233 \pm 0.031$ & $0.3233 \pm 0.031$ & 0.0000 & -0.0000 \\
$-0.3333 \pm 0.0402$ & $0.3333 \pm 0.0402$ & & \\
$-0.0680 \pm 0.0446$ & $0.0680 \pm 0.0446$ & $0.3067 \pm 0.0210$ & $-0.3067 \pm 0.0210$ \\
$0.2209 \pm 0.0528$ & $-0.2209 \pm 0.0528$ & & \\
0.1667 & 0.1667 & 0.1667 & 0.1667 \\
0.1667 & 0.1667 & & \\
& & &
\end{tabular}

Note:-Data are means. mapping multiple vascular territories by using $\mathrm{x}$ and $\mathrm{y}$ gradients to encode arteries within the labeling plane. ${ }^{15}$ It has been applied in the evaluation of collateral circulation in ischemic cerebrovascular diseases in previous studies. ${ }^{16,17}$

In this study, we applied VE-PCASL in patients with intracranial AVMs to identify feeding arteries of AVMs on the basis of the estimated supply fractions of the arteries. Due to the presence of noise in the supply fraction maps and potential contamination of ASL signals between vessels, a cutoff or threshold was applied on the estimated supply fraction to identify the "true" feeding arteries of AVMs. The accuracy of VE-PCASL for identifying feeding arteries at various cutoff values of the supply fraction was evaluated by using DSA as the criterion standard.

\section{MATERIALS AND METHODS \\ Patients}

This study was approved by the institutional review boards, and written informed consent was obtained from all participants. The inclusion criteria were patients diagnosed with intracranial AVMs and without other brain lesions who were scheduled for DSA examination at Beijing Tiantan Hospital. The general contraindications for MR imaging examination were applied as the exclusion criteria.

\section{DSA Technique}

DSA was performed according to a standard protocol during routine clinical examinations on a biplane angiography system (Advantx LCV +; GE Healthcare, Hertfordshire, UK). A 5F diagnostic catheter was navigated into the bilateral internal carotid, external carotid, and vertebral arteries (VAs) (6 vessels), respectively, via the right femoral artery to acquire standard anteroposterior and lateral projections, each by manual delivery of a 5- to 7-mL iodinated contrast agent injection per run (iodixanol, Visipaque; GE Healthcare, Co. Cork, Ireland).

\section{MR Imaging}

MR imaging was performed on a $3 \mathrm{~T}$ Tim Trio system (Siemens, Erlangen, Germany) by using a body coil transmitter and a 12channel head coil as a receiver. First, a sagittal localizer scan was performed for anatomic reference; then TOF MRA was performed to select the right and left ICAs and vertebral arteries, on the basis of which the tagging plane was determined (Fig 1). The labeling offset from the center of the imaging slab was $80 \mathrm{~mm}$. In this axial tagging plane, the ICAs and vertebral arteries had an approximately trapezoidal arrangement and the direction of flow was predominantly inferior-to-superior. The image sections were positioned parallel to the labeling plane. Postlabeling delay (PLD) was set at 3 time points (1, 1.3, and 1.6 seconds) in 6 patients and at a single time point of 1 second in the remaining 12 patients. Imaging parameters were as follows: TR/TE/flip angle $=3000 \mathrm{~ms} / 10$ $\mathrm{ms} / 90^{\circ}$ for $\mathrm{PLD}=1$ second $($ while $\mathrm{TR}=$ 3.3 seconds for PLD $=1.3$ seconds and $\mathrm{TR}=3.6$ seconds for $\mathrm{PLD}=1.6$ seconds), FOV $=220 \times 220 \mathrm{~mm}^{2}, 12$ sections with a 6-mm thickness and 1.5-mm gap, matrix 
size $=64 \times 64,7 / 8$ partial $k$-space, 120 measurements, and 2D EPI readout. We performed 6 cycles of VE-PCASL: global tag, control, tag of the left-versus-right ICA, tag of ICA versus the vertebrobasilar artery. ${ }^{15}$ The total scanning time was 6.2 minutes for 1 PLD and 20 minutes for 3 PLDs.

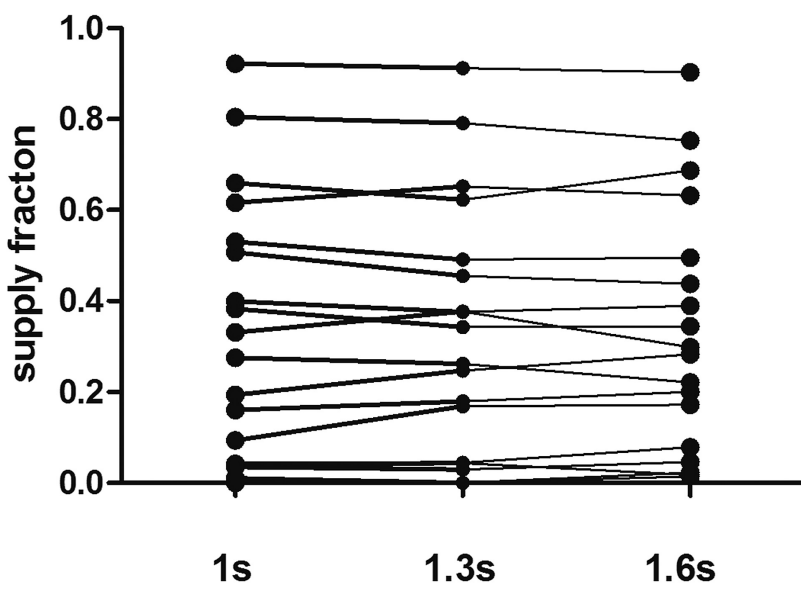

FIG 2. For the 6 patients (18 vessels) with 3 PLDs, there are no significant differences in the supply fractions with relative labeling efficiencies $(P>.05)$.

Table 2: Demographic data on the studied population and characterization information of AVMs

\begin{tabular}{|c|c|c|c|c|c|c|}
\hline \multirow[b]{2}{*}{ Patient No. } & \multirow[b]{2}{*}{ Age (yr) } & \multirow[b]{2}{*}{ Sex } & \multirow{2}{*}{$\begin{array}{l}\text { Spetzler-Martin } \\
\text { Classification }\end{array}$} & \multicolumn{3}{|c|}{$\begin{array}{l}\text { Major Feeding Arteries } \\
\text { Diagnosed by DSA }\end{array}$} \\
\hline & & & & RICA & LICA & VA \\
\hline 1 & 23 & $M$ & S2EIVO & 0 & 1 & 1 \\
\hline 2 & 21 & $M$ & S2EOV1 & 1 & 1 & 1 \\
\hline 3 & 34 & $M$ & S2EOVO & 0 & 1 & 1 \\
\hline 4 & 44 & $M$ & S2E1V1 & 1 & 1 & 1 \\
\hline 5 & 27 & $\mathrm{~F}$ & S3EIVO & 1 & 0 & 1 \\
\hline 6 & 22 & $\mathrm{~F}$ & S3E1V1 & 1 & 1 & 1 \\
\hline $7^{a}$ & 40 & $M$ & S2EOVO & 0 & 1 & 1 \\
\hline $8^{a}$ & 46 & $\mathrm{~F}$ & S2EOVO & 1 & 1 & 1 \\
\hline $9^{a}$ & 31 & $\mathrm{~F}$ & S2EIVO & 1 & 0 & 1 \\
\hline $10^{a}$ & 47 & $\mathrm{~F}$ & S2EIVO & 1 & 0 & 1 \\
\hline $11^{\mathrm{a}}$ & 13 & $M$ & SIEOVO & 0 & 1 & 1 \\
\hline $12^{\mathrm{a}}$ & 39 & $M$ & S2EOVO & 1 & 0 & 0 \\
\hline 13 & 27 & $\mathrm{~F}$ & S2EIVO & 1 & 0 & 1 \\
\hline 14 & 31 & $M$ & S3EIVO & 1 & 1 & 1 \\
\hline 15 & 25 & $M$ & SIEIVO & 0 & 1 & 1 \\
\hline 16 & 47 & $M$ & S2EOVO & 1 & 0 & 1 \\
\hline 17 & 39 & $\mathrm{~F}$ & SIEIV1 & 0 & 1 & 1 \\
\hline 18 & 28 & $\mathrm{~F}$ & SIEOVO & 0 & 1 & 0 \\
\hline
\end{tabular}

Note: $-S$ indicates the size of the cerebral AVM nidus $(1,<3 \mathrm{~cm} ; 2,3 \sim 6 \mathrm{~cm} ; 3,>6 \mathrm{~cm})$; E, eloquence of adjacent brain ( 0 , noneloquent; 1 , eloquent); $\mathrm{V}$, venous drainage pattern ( 0 , superficial draining vein; 1 , deep draining vein).

a Patients who had 3 PLD time points. Zero and 1 indicate arteries that have or have not contributed to AVM lesions.

Table 3: Different cutoff values of the supply fraction of feeding arteries of the AVM and the resulting sensitivity and specificity with standard or relative labeling efficiencies respectively

\begin{tabular}{lccccc}
\hline & \multicolumn{2}{c}{ Standard } & & \multicolumn{2}{c}{ Relative } \\
\cline { 2 - 3 } \cline { 5 - 6 } Cutoff Value & Sensitivity & Specificity & & Sensitivity & Specificity \\
\hline $0 \%$ & $100 \%$ & 0 & $100 \%$ & $6.67 \%$ \\
$5 \%$ & $97.44 \%$ & $40 \%$ & & $97.44 \%$ & $66.67 \%$ \\
$10 \%$ & $92.31 \%$ & $73.33 \%$ & & $89.74 \%$ & $80 \%$ \\
$15 \%$ & $84.62 \%$ & $93.33 \%$ & & $82.05 \%$ & $93.33 \%$ \\
$20 \%$ & $76.92 \%$ & $93.33 \%$ & & $79.49 \%$ & $93.33 \%$ \\
$25 \%$ & $74.36 \%$ & $93.33 \%$ & $69.23 \%$ & $93.33 \%$ \\
\hline
\end{tabular}

\section{Data Processing}

EPI images were corrected for motion. Global perfusionmeasure the mean value and SD. Basal ganglia signal (mean) + 8 SDs was set as the threshold to generate a mask for the AVM esion on the global perfusion image. ${ }^{12}$ cies ${ }^{15}$ respectively. The standard tagging efficiency matrix was adopted from previous studies. ${ }^{15}$ In addition, the relative tagging efficiency for each artery (assuming that the efficiency of nonselective labeling is 1) was measured directly from the experimental data by using the histogram method in each subject. ${ }^{19}$ The estimated relative tagging efficiencies were then used in the construction of the modified Hadamard encoding atrix (Table 1).

The AVM mask determined on global perfusion images was overlaid on individual perfusion maps of the 3 main feeding arteries. The contributions of the 3 main arteries to the AVM lesion were quantified by calculating supply fractions. ${ }^{19}$ The supply fraction was defined as CBF values of all voxels within the AVM mask on individual $\mathrm{CBF}$ maps $\left(\mathrm{CBF}_{\text {ind }}\right)$ divided by the summation of $\mathrm{CBF}$ values of the 3 main arteries-that is, $\mathrm{CBF}_{\text {ind }}$ / $\left(\mathrm{CBF}_{\mathrm{LICA}}+\mathrm{CBF}_{\mathrm{RICA}}+\mathrm{CBF}_{\mathrm{VA}}\right) \times$ $100 \%$.

\section{Analysis}

Two experienced neurosurgeons (both with $>10$ years' experience) who were blinded to the results of VE-PCASL reviewed DSAs in a random order to identify the major arteries that supplied the AVM lesions. In each patient, each of the 3 major arteries (a total of 54 arteries) was labeled zero or 1 as feeding arteries of the AVM. In cases of disagreement, a consensus was sought. Two experienced researchers reviewed the ASL perfusionweighted images independently in a random order. Image quality was scored from 5 to 1 , corresponding to excellent, good, fair, poor, or uninterpretable, respectively. The Cohen $\kappa$ was obtained to evaluate interobserver agreement on image quality. The $\kappa$ coefficient of agreement was graded as follows: $0-0.2=$ poor to slight; $0.21-0.4=$ fair; $0.41-$ $0.6=$ moderate; $0.61-0.8=$ excellent; 0.81-0.99 = nearly perfect; $1.0=$ perfect. $^{20}$ 
ROC Curve

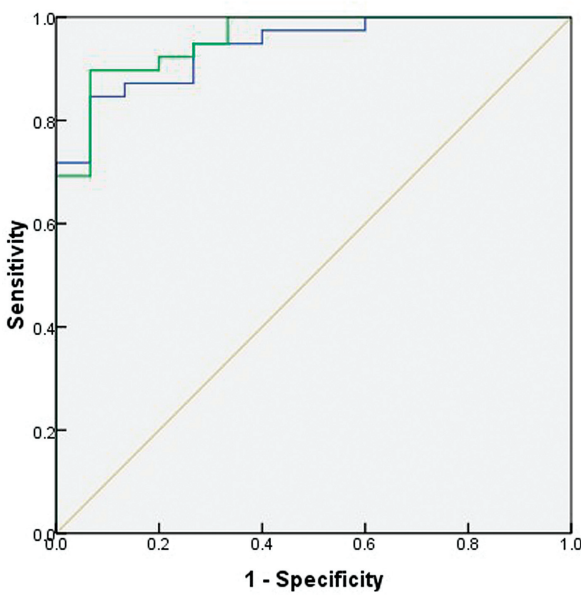

Source of the Curve - standard

lative

- Reference Line

FIG 3. ROC curves of VE-PCASL with standard (blue line) and relative (green line) labeling efficiencies, respectively, for the identification of feeding arteries, and the AUC is larger for relative labeling efficiencies.
Receiver operating characteristic analysis (ROC) curves were generated from the supply fractions of the 3 major arteries obtained by VE-PCASL with PLD = 1 second, by using the Statistical Package for the Social Sciences, Version 16.0 (IBM, Armonk, New York). The area under the ROC curve (AUC) was calculated to compare the diagnostic accuracy of VE-PCASL with standard and relative labeling efficiencies, respectively. The cutoff of the supply fraction was determined as the value that achieved a targeted balance between sensitivity and specificity. In the present study, the value of the supply fraction associated with the maximal sum of sensitivity and specificity was chosen as the optimal cutoff.

Statistical analyses were performed by using within-subject ANOVA to compare supply fractions acquired with different PLDs. The data processing was performed with Matlab R2009a (MathWorks, Natick, Massachusetts).

\section{RESULTS}

\section{Patients}

Between December 2011 and November 2012, eighteen consecutive patients ( 8 women/10 men; mean age, $32.8 \pm 10.8$ years) with intracranial AVMs were recruited for this prospective study. All 18 patients demonstrated no other lesions in the brain, and 1

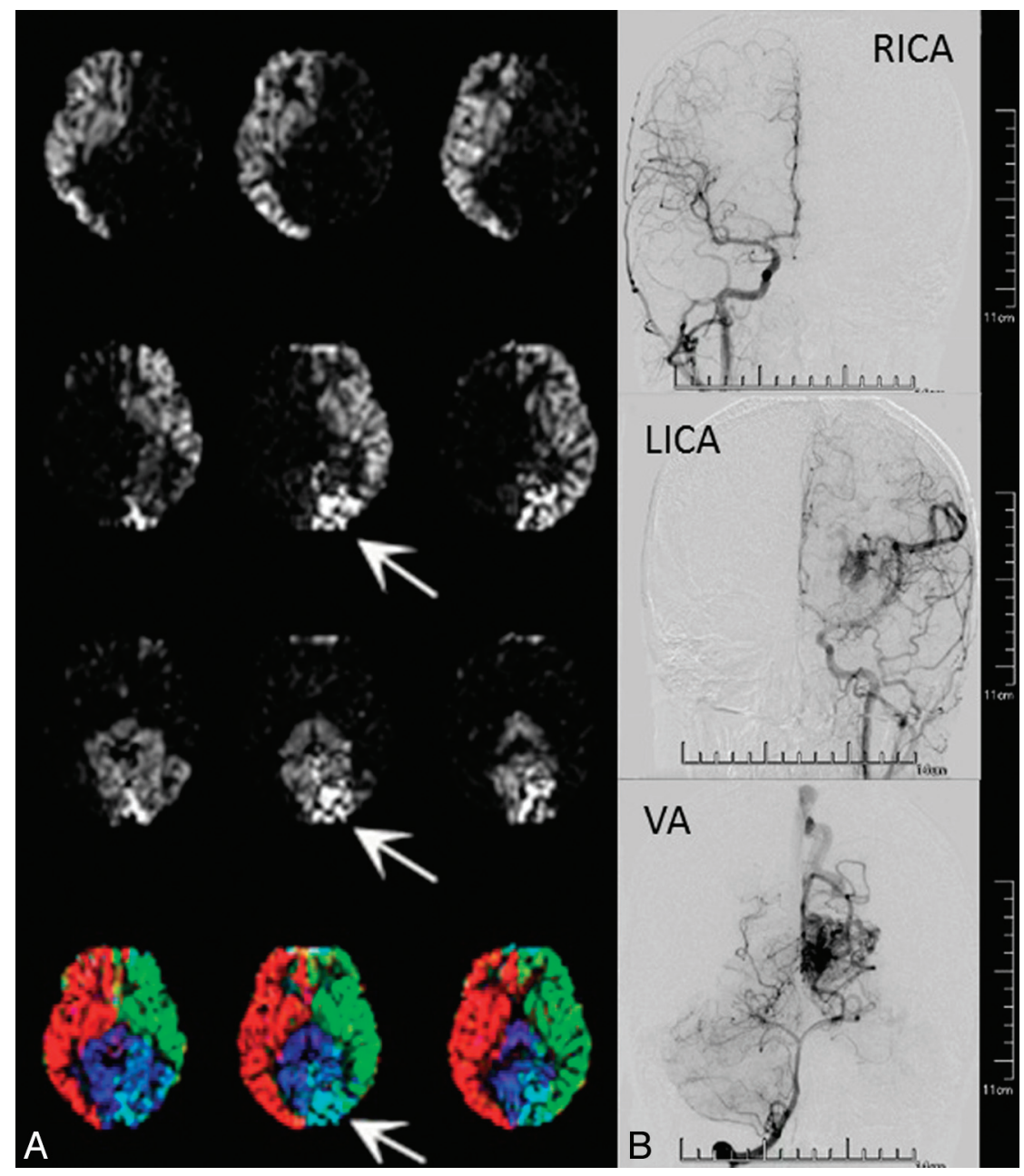

FIG 4. Patient 1 , a 23 -year-old man. He had headaches and blurred vision in both eyes for half a year. The supply fractions of the left ICA, VA, and right ICA were $52.59 \%, 46.75 \%, 0.66 \%$ and $49.27 \%, 50.71 \%, 0$ with standard and relative labeling efficiencies, respectively, showing good agreement between the 2 methods. A, VE-PCASL with standard labeling efficiency (arrows show the AVM lesion). B, DSA shows that the AVM lesion is fed by the left ICA and VA. 


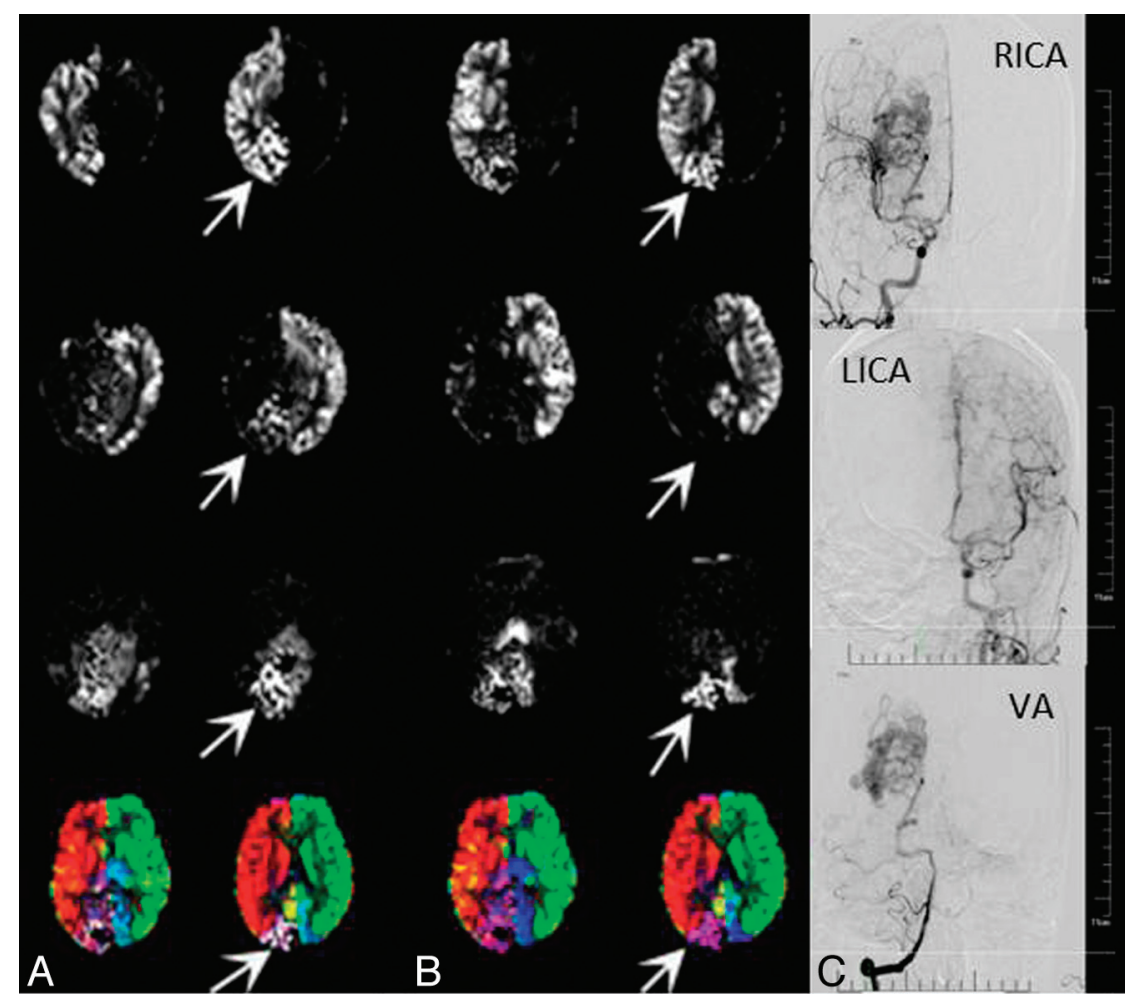

FIG 5. Patient 13, a 27-year-old woman. She had a sudden severe headache accompanying a visual field defect 2 months ago, which was confirmed as spontaneous intraventricular hemorrhage by CT. A, VE-PCASL with standard labeling efficiencies shows relatively poor separation of left-versus-right ICA perfusion territories, and there are abnormally high signals in the left ICA perfusion territory with the supply fraction of $14.31 \%$, mismatching the results of DSA. B, VE-PCASL with relative labeling efficiencies shows an improved separation, and there are fewer abnormally high signals from the left ICA perfusion territory with the supply fraction of $1.14 \%$ (arrows show the AVM lesion). C, DSA shows that the AVM lesion is fed by the VA and right ICA.

patient had an interventional operation to partially embolize the AVM lesion. The demographic data of the studied population are listed in Table 2. The time interval between preoperative DSA and VE-PCASL was $2 \sim 40$ days (mean $=11.7 \pm 14.1$ days).

\section{Image Quality}

The identification of feeding arteries of the AVM was consistent between the 2 neurosurgeons on the basis of DSA reports. VEPCASL was performed successfully in all 18 patients in whom AVM lesions could be identified with obviously high CBF values. The average score of image quality of the ASL maps was $4.36 \pm$ 0.72 . The $\kappa$ coefficient was 0.723 between the 2 raters, which indicates excellent agreement.

\section{Reliability of VE-PCASL}

Supply fractions of individual feeding arteries to the AVM lesions were successfully obtained by using both standard and relative tagging efficiencies, respectively. For the 6 patients with 3 PLDs, there were no significant differences in the supply fractions obtained at 3 PLDs $(P>.05$, Fig 2$)$.

\section{Diagnostic Accuracy of VE-PCASL}

The diagnostic accuracy of VE-PCASL assessed by ROC showed high sensitivity and specificity at various cutoff levels for both standard and relative labeling efficiencies (Table 3). Both methods yielded a high diagnostic accuracy with an AUC $>0.9(P<$ .01) (Fig 3).
For VE-PCASL encoded with standard labeling efficiencies, the ROC curve showed that the cutoff of $15.17 \%$ supply fraction is optimal for identifying feeding arteries to the AVM. For that cutoff level (ie, supply fraction $\geq 15.17 \%$ ), the sensitivity was $84.62 \%$ and the specificity was $93.33 \%$, the positive predictive value was $97.06 \%$, and the negative predictive value was $70 \%$. The AUC was $0.942(P<.01)$, indicating a high level of accuracy (Fig 4).

VE-PCASL with relative labeling efficiencies showed an improved ability to discriminate ASL signals from different arteries. This resulted in an improved sensitivity while maintaining the specificity with a decreased cutoff of supply fractions. The AUC for this method was 0.957 , and the optimal cutoff of the supply fraction was $11.73 \%$, which led to an $89.74 \%$ sensitivity, $93.33 \%$ specificity, $97.22 \%$ positive predictive value, and $77.78 \%$ negative predictive value. The AUC by using relative labeling efficiencies was greater than that of standard labeling efficiencies, though there were no significant differences between the AUCs of the 2 decoding methods (Fig 5).

However, VE-PCASL with relative labeling efficiencies was not perfect. Figure 6 shows an AVM located in the right occipital lobe that was fed by the VA and right ICA on DSA. VE-PCASL with standard labeling efficiencies showed abnormally high signals in the left ICA perfusion territory with a supply fraction of $27.53 \%$, mismatching the results of DSA. VE-PCASL with relative labeling efficiencies reduced the supply fraction of the left ICA to 


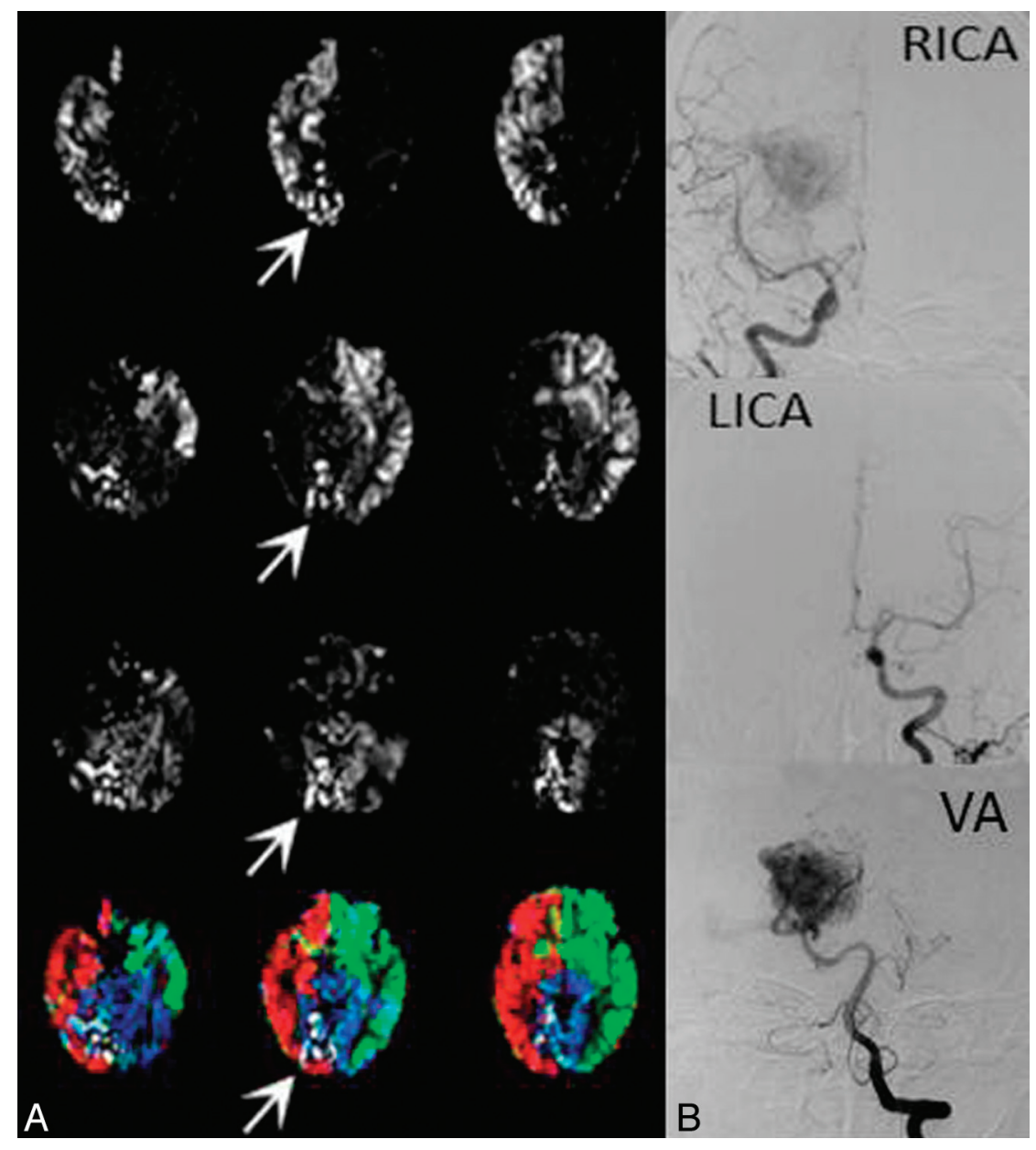

FIG 6. Patient 9, a 31-year-old woman. She had dizziness for approximately 2 years. VE-PCASL with standard labeling efficiencies showed abnormally high signals in the left ICA perfusion territory with the supply fraction of $27.53 \%$, mismatching the results of DSA. A, VE-PCASL with relative labeling efficiencies shows reduced abnormal signals with the supply fraction of $25.67 \%$ but still fails to match the results of DSA (arrows show the AVM lesion). B, DSA shows that the AVM lesion is fed by the VA and right ICA.

$25.67 \%$ but still failed to match the results of DSA. There were still hyperintensities in the left ICA perfusion territory.

\section{DISCUSSION}

VE-PCASL is a recently introduced time-efficient method for mapping multiple vascular territories. It can estimate the fraction of CBF supplied by each artery by using $x-y$ gradients to encode the major arteries within the labeling plane. The imaging process is conceptually similar to that of conventional angiography without the use of $\mathrm{x}$-rays or iodinated contrast agents. As a result, VE-PCASL has engendered increasing attention in the evaluation of cerebrovascular disorders such as steno-occlusive disease, aneurysms, and AVMs. However, 1 shortcoming of VE-PCASL is its sensitivity to magnetic field inhomogeneity effects at the labeling location, which affects tagging efficiencies of individual arteries. ${ }^{21}$ Furthermore, for AVMs, there is clearance of tagged blood by venous flow due to the lack of a normal capillary network, which would lead to inaccuracy in quantifying CBF. In the present study, we estimated the relative tagging efficiencies of 3 major arteries in each individual patient by using the histogram method. We also applied 3 different PLDs to check whether the supply fraction can be estimated reliably. The results were relatively stable across different PLDs; this finding indicates that VE-PCASL can provide a reliable estimation of supply fractions of individual arteries despite several potential confounding factors such as relaxation and venous outflow of the labeled blood.

Optimal Hadamard encoding assumes that all inflowing blood is either fully inverted or fully relaxed for each tagging cycle, and there are equal numbers of tag and control conditions for each vessel. ${ }^{15}$ This assumption may not be realistic because of vessel geometry and variations in tagging efficiencies due to field inhomogeneity effects. As a result, the discrimination of arteries may not be perfectly achieved and there may be contamination of ASL signals from different arteries. This result may not be prominent in healthy populations but would be noteworthy in AVM lesions in which $\mathrm{CBF}$ is abnormally high. To circumvent this problem, Wong ${ }^{15}$ proposed a histogram-based method for estimating vessel-specific labeling efficiencies from VE-PCASL data, which were applied in the construction of the encoding matrices to improve the separation of supplying arteries within vascular territories.

Overall, VE-PCASL with both standard and relative labeling efficiencies showed a high level of accuracy in identifying feeding arteries (AUC $=0.942$ and 0.957). In the present study, the optimal cutoff was chosen as a balance between sensitivity and specificity (maximal sum). Because no statistical significance was observed, it is too 
soon to conclude that VE-PCASL with relative labeling efficiencies has better performance compared with that of standard labeling efficiencies. VE-PCASL with both standard and relative labeling efficiencies awaits further evaluation in larger scale clinical studies.

Various MR imaging techniques have been applied as noninvasive and radiation-free methods for the evaluation of intracranial AVMs. However, the subjective interpretation of feeding arteries may be viewer-dependent. VE-PCASL evaluates feeding arteries and AVM lesions differently than TOF-MRA or DSA. It does not display the vascular architecture directly but provides a quantitative estimate of supply fractions of individual arteries, which can be displayed in color scales. The fractional supply was calculated to evaluate the role of each feeding artery quantitatively rather than by qualitative approaches without standardization. In addition, it can help to determine dominant feeding arteries, the pattern of which would influence the chances for complete or subtotal obliteration of AVM lesions. ${ }^{22}$ The cutoff or threshold of the supply fraction should be determined according to the clinical purpose. For example, if the purpose was for preliminary screening of feeding arteries, the lower cutoff (higher sensitivity) should be applied, while a higher cutoff (higher specificity) should be chosen for precise identification of feeding arteries.

In addition to the advantages of a quantitative evaluation of supply fractions, VE-PCASL can display the spatial relationship between AVM lesions and functional or eloquent cortices, which would provide valuable information for neurosurgical operations. Furthermore, VE-PCASL can evaluate hypoperfused areas around the AVM to probe a potential "steal phenomenon" related to the AVM lesion. ${ }^{12}$ Recently, VE-PCASL has also been expanded to encode branches of the circle of Willis, even without prior knowledge of vessel positions. ${ }^{23}$

A limitation of our study is that the number of patients was relatively small; thus, the selection of the cutoff may serve as a reference rather than a criterion. Another limitation is that we did not label bilateral external carotid arteries with VE-PCASL, which may also contribute to the feeding of intracranial AVMs (1 AVM in this study was confirmed to be fed partially by ipsilateral external carotid artery on DSA). However the latter problem has recently been resolved technically ${ }^{17}$ and labeling bilateral external carotid arteries can be applied in assessments of AVM lesions in the future.

\section{CONCLUSIONS}

Quantitative supply fractions of feeding arteries to intracranial AVMs can be reliably estimated with VE-PCASL. VE-PCASL with either standard or relative labeling efficiencies demonstrated a high level of accuracy for identifying feeding arteries of AVMs. Even though VE-PCASL cannot replace DSA for clinical diagnosis currently, it can act as preoperative adjunct to estimate relative contributions of feeding arteries.

Disclosures: Rong Xue-RELATED: Grant: Ministry of Science and Technology of China, ${ }^{*}$ Chinese Academy of Sciences Knowledge Innovation Project.* Danny J.J. Wang-RELATED: Grant: National Institutes of Health. *Money paid to the institution.

\section{REFERENCES}

1. Hernesniemi JA, Dashti R, Juvela S, et al. Natural history of brain arteriovenous malformations: a long-term follow-up study of risk of hemorrhage in 238 patients. Neurosurgery 2008;63:823-29, discussion 829-31

2. Pik JH, Morgan MK. Microsurgery for small arteriovenous malformations of the brain: results in 110 consecutive patients. Neurosurgery 2000;47:571-75, discussion 575-77

3. Klimo P Jr, Rao G, Brockmeyer D. Pediatric arteriovenous malformations: a 15-year experience with an emphasis on residual and recurrent lesions. Childs Nerv Syst 2007;23:31-37

4. van Beijnum J, van der Worp HB, Buis DR, et al. Treatment of brain arteriovenous malformations: a systematic review and meta-analysis. JAMA 2011;306:2011-19

5. Spetzler RF, Martin NA, Carter LP, et al. Surgical management of large AVM's by staged embolization and operative excision. $J \mathrm{Neu}$ rosurg 1987;67:17-28

6. Yarsargil MG, Curcic M, Kis M, et al. Microneurosurgery (IIIB). Stuttgart, Germany and New York: Thieme; 1988

7. Willinsky RA, Taylor SM, TerBrugge K, et al. Neurologic complications of cerebral angiography: prospective analysis of 2,899 procedures and review of the literature. Radiology 2003;227:522-28

8. Hankey GJ, Warlow CP, Sellar RJ. Cerebral angiographic risk in mild cerebrovascular disease. Stroke 1990;21:209-22

9. Hadizadeh DR, von Falkenhausen M, Gieseke J, et al. Cerebral arteriovenous malformation: Spetzler-Martin classification at subsecond-temporal-resolution four-dimensional MR angiography compared with that at DSA. Radiology 2008;246:205-13

10. Yu S, Yan L, Yao Y, et al. Noncontrast dynamic MRA in intracranial arteriovenous malformation (AVM): comparison with time of flight (TOF) and digital subtraction angiography (DSA). Magn Reson Imaging 2012;30:869-77

11. Detre JA, Leigh JS, Williams DS, et al. Perfusion imaging. Magn Reson Med 1992;23:37-45

12. Wolf RL, Wang J, Detre JA, et al. Arteriovenous shunt visualization in arteriovenous malformations with arterial spin-labeling MR imaging. AJNR Am J Neuroradiol 2008;29:681-87

13. Pollock JM, Whitlow CT, Simonds J, et al. Response of arteriovenous malformations to gamma knife therapy evaluated with pulsed arterial spin-labeling MRI perfusion. AJR Am J Roentgenol 2011;196: 15-22

14. Wu WC, Fernandez-Seara M, Detre JA, et al. A theoretical and experimental investigation of the tagging efficiency of pseudocontinuous arterial spin labeling. Magn Reson Med 2007;58:1020-27

15. Wong EC. Vessel-encoded arterial spin-labeling using pseudocontinuous tagging. Magn Reson Med 2007;58:1086-91

16. Wu B, Wang X, Guo J, et al. Collateral circulation imaging: MR perfusion territory arterial spin-labeling at 3T. AJNR Am J Neuroradiol 2008;29:1855-60

17. Dang Y, Wu B, Sun Y, et al. Quantitative assessment of external carotid artery territory supply with modified vessel-encoded arterial spin-labeling. AJNR Am J Neuroradiol 2012;33:1380-86

18. Wang J, Alsop DC, Li L, et al. Comparison of quantitative perfusion imaging using arterial spin labeling at 1.5 and 4.0 Tesla. Magn Reson Med 2002;48:242-54

19. Kansagra AP, Wong EC. Quantitative assessment of mixed cerebral vascular territory supply with vessel encoded arterial spin labeling MRI. Stroke 2008;39:2980-85

20. Landis JR, Koch GG. The measurement of observer agreement for categorical data. Biometrics 1977;33:159-74

21. Wong EC. Quantifying CBF with pulsed ASL: technical and pulse sequence factors. J Magn Reson Imaging 2005;22:727-31

22. Cohadon F. Dolenc VV, Lobo Antunes J, et al. Advances and Technical Standards in Neurosurgery. (vol. 24). New York: Springer-Verlag; 1998

23. Wong EC, Guo J. Blind detection of vascular sources and territories using random vessel encoded arterial spin labeling. MAGMA 2012; 25:95-101 